\title{
Directional Antenna for Multipath Ad Hoc Routing
}

\author{
Hamid Gharavi and Bin $\mathrm{Hu}$ \\ National Institute of Standards and Technology \\ 100 Bureau Drive, Stop 8920, Gaithersburg, MD 20899-8920, USA \\ E-mail: [gharavi, bhu]@nist.gov
}

\begin{abstract}
Multipath routing can be effectively used to maximize the utilization of network resources for real-time traffic. In mobile ad-hoc network environments, this approach can suffer from co-channel interference due to the simultaneous transmission of packets via multiple routes. In this paper, by exploiting the directionality of directional antennas, a Directional Dynamic Source Routing (DDSR)-based Multipath routing protocol is proposed for mobile wireless ad hoc networks. Three metrics are considered in the route discovery process in order to select the best multiple routes. These are hop count, beam overlaps, and the number of joint nodes between the different routes. The proposed directional routing protocol has shown to be capable of minimizing inter-path and intra-path interference, hence significantly improving the system throughput performance for simultaneous transmission of multimedia information over multiple routes.
\end{abstract}

Index Terms-Ad hoc networks, routing protocols, DSR, directional antennas, directional routing, multipath routing.

\section{INTRODUCTION}

The major obstacle in supporting real-time traffic for MANET is the long delays associated with frequent route changes due to the dynamically changing network topology. This includes latency in detecting a link loss, as well as the time needed to discover a new route. Multipath routing has been shown to be an effective approach to reduce the blocking capabilities for multimedia traffic. This approach has received considerable attention in recent years [1], [2], [3], [4], [5], [6], [7], [8]. However, in contrast to wired IP networks, in mobile ad-hoc network environments, multiple routing protocols can suffer from co-channel interference due to the simultaneous transmission of packets via multiple routes. This is mainly due to the fact that in such environments, nodes in different routes are normally within interference range of each other. The suppression of interference between nodes in different routes (inter-path interference) has been considered previously, using a multi-channel approach [6]. In this approach, omni-directional antenna was applied. Alternatively, by using directional antenna it is possible to suppress inter-path and intra-path interferences for multiple path routing and single path routing, respectively.

For single path routing, smart antenna techniques have been extensively investigated in order to improve the achievable performance and system capacity. They are capable of providing spatial reuse, longer ranges, interference suppression, and other beneficial features [9], [10], [11], [12]. In [9], a brief overview of smart antenna techniques was provided and the issues that arose when applying these techniques in ad hoc networks were then described. Yi et al. [10] provided a theoretical framework to understand how much capacity improvement can be achieved using directional antennas. Aimed at developing a complete ad hoc networking system with directional antennas, including the unique challenge of real-life prototype development and experimentation, Ramanathan [11] proposed utilizing directional antennas for ad hoc networking (UDAAN). Based on neighbor discovery [11], Choudhury [12] proposed MultiHop RTS MAC (MMAC), which uses multihop RTSs to establish links between distant nodes, and then transmit CTS, DATA, and ACK over a single hop. Takai proposed a novel carrier sensing mechanism called Directional Virtual Carrier Sensing (DVCS) for wireless communication using directional antennas in [13], which only needs information on Angle of Arrival (AOA) and antenna gain for each signal from the underlying physical device. Along a different avenue, much attention has also been paid to exploiting the spatial diversity of antenna arrays. Park [14] designed a novel MAC protocol, Mitigating Interference using Multiple Antennas with Antenna Selection (MIMA/AS-MAC), for MIMO aided ad hoc wireless networks. The authors in [7] did a comparative study on the performance of multipath routing using omni-directional and directional antenna. The simulation results in [7] showed that directional antenna is capable of providing significant improvement to the performance of multipath routing. However, the employed directional antennabased multipath routing protocol did not aim at suppressing the inter-path and intra-path interferences. In this paper we present a DDSR-based multipath routing protocol for wireless ad-hoc networks by exploiting the directionality of directional antennas to suppress both inter-path and intra-path interferences.

This paper is organized as follows: In Section II, after a brief introduction to the DSR routing protocol, we propose a DDSRbased multipath routing protocol. In Section III the attainable performance of the proposed DDSR-based multipath routing protocol is investigated comparatively with other routing protocols. Finally, we offer our conclusions in Section IV.

\section{Directional DSR-BASEd Multipath Routing PROTOCOL}

In this section, DDSR-based multipath routing protocol invoked for wireless ad hoc networks is described. In this approach, multiple paths from the source node to the destination node are selected according to hop count, overlap count and the number of joint nodes between the different routes. 
DSR [15] is a source-initiated on-demand routing protocols, where complete hop-by-hop routes are stored in route cache of each node. When a source node attempts to send a data packet to an unknown destination, it initiates a route discovery process to find routes to the destination node. In a route discovery process, Route Request (RREQ) packets and Route Reply (RREP) packets are used to set up the routes from the source node to the destination node. Once the source node finds routes to the destination, it inserts the source route to the data packets and send them out. If a link break occurs while the route is active, Route Error (RERR) messages are generated. When a RRER is received, the receiving node removes the hop in error from its route cache and truncates all routes containing this hop [15].

\section{A. Route Discovery}

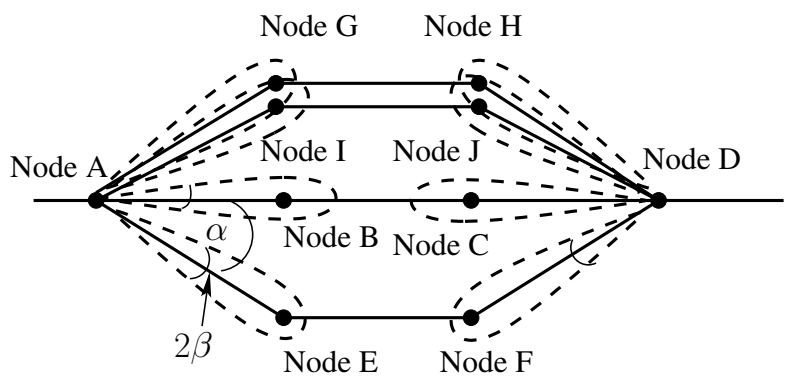

Fig. 1. An example where the beam of $\mathrm{A} \rightarrow \mathrm{E}$ does not overlap with the beam of $\mathrm{D} \rightarrow \mathrm{F}$, since we have $\alpha>\beta$.

We consider overlap count as an important metric in the proposed DDSR-based multipath routing protocol. This is based on the number of overlaps among beams over multiple paths in the route discovery process, so that multiple routes with the fewest overlaps can be selected. As shown in Fig. 1, when directional antennas are employed, the transmit beam of $\mathrm{A} \rightarrow \mathrm{E}$ does not overlap with the receive beam of $\mathrm{D} \rightarrow \mathrm{F}$. This indicates that the transmission from node $\mathrm{A}$ to node $\mathrm{E}$ does not impact node $\mathrm{D}$. By contrast, the transmit beam of $\mathrm{A} \rightarrow \mathrm{B}$ overlaps with the receive beam of $\mathrm{D} \rightarrow \mathrm{C}$, meaning that the transmission from node $\mathrm{A}$ to node $\mathrm{B}$ interferes with node $\mathrm{D}$. Note that in a wireless multi-hop network, the interference from the nodes hops away may degrade the throughput greatly [16], [17]. Hence, in this case the route of $\mathrm{A} \rightarrow \mathrm{E} \rightarrow \mathrm{F} \rightarrow \mathrm{D}$ is better than that of $\mathrm{A} \rightarrow \mathrm{B} \rightarrow \mathrm{C} \rightarrow \mathrm{D}$.

In the proposed routing protocol, if the angle between $\mathrm{A} \rightarrow \mathrm{B}$ and $\mathrm{A} \rightarrow \mathrm{D}$ is less than a threshold $\beta$ while the angle between $\mathrm{D} \rightarrow \mathrm{C}$ and $\mathrm{D} \rightarrow \mathrm{A}$ is also less than the threshold $\beta$, they overlap and hence interfere with each other. Therefore, in order to prevent any overlap, a sharp beam with a beam-width of $2 \beta$ degree should be used, where

$$
\alpha>\beta \text {. }
$$

As we can observe from Fig. 1, there is no overlap in route $\mathrm{A} \rightarrow \mathrm{G} \rightarrow \mathrm{H} \rightarrow \mathrm{D}$ or route $\mathrm{A} \rightarrow \mathrm{I} \rightarrow \mathrm{J} \rightarrow \mathrm{D}$. Under normal conditions (single-path DSR-based routing), any one of these routes can be selected for data transmission. It should be noted however, that in single-path DDSR directional routing only intra-path overlaps need to be counted in order to eliminate interferences between neighboring hopping nodes [18]. Thus, in the case of multipath schemes, routes $\mathrm{A} \rightarrow \mathrm{G} \rightarrow \mathrm{H} \rightarrow \mathrm{D}$ and $\mathrm{A} \rightarrow \mathrm{I} \rightarrow \mathrm{J} \rightarrow \mathrm{D}$ can not be simultaneously selected to transmitting packets because of the inter-path interferences between them. For example, the transmit beam of $\mathrm{A} \rightarrow \mathrm{G}$ overlaps with the receive beam of $\mathrm{I} \rightarrow \mathrm{A}$ while the transmit beam of $\mathrm{A} \rightarrow \mathrm{I}$ overlaps with the receive beam of $\mathrm{G} \rightarrow \mathrm{A}$. Instead, routes $\mathrm{A} \rightarrow \mathrm{E} \rightarrow \mathrm{F} \rightarrow \mathrm{D}$ and $\mathrm{A} \rightarrow \mathrm{G} \rightarrow \mathrm{H} \rightarrow \mathrm{D}$, or routes $\mathrm{A} \rightarrow \mathrm{E} \rightarrow \mathrm{F} \rightarrow \mathrm{D}$ and $\mathrm{A} \rightarrow \mathrm{I} \rightarrow \mathrm{J} \rightarrow \mathrm{D}$ can be used for simultaneous packet transmission. Under these conditions there would be no intra-path or inter-path interference.

In the route discovery process of the proposed DDSR-based multi-path routing protocol, the transmitting node will insert its address and position information into the received RREQ or RREP and then forwarded it to the neighboring nodes or the next node. Under these conditions, every node uses its own positional information as well as the positional information that the node has received from its neighboring node (via RREQ and RREP) to calculate the direction of arrival (DOA). According to the position information, the DOAs and $\beta$ in (1), the intrapath overlap count of a specific route can be achieved. Note that for two adjacent hops, i.e. the hops of $\mathrm{A} \& \mathrm{E}$ and $\mathrm{E} \& \mathrm{~F}$ in $\mathrm{A} \rightarrow \mathrm{E} \rightarrow \mathrm{F} \rightarrow \mathrm{D}$, although they don't overlap with each other, packet loss will inevitably occur when they transmit and receive data at same time. This is mainly due to the fact that a packet sent by node A cannot be received by node $\mathrm{E}$, while this node itself is engaged in relaying a packet to node F. Obviously, this kind of packet loss is due to a form of hidden terminal effect, which is unique in the case of directional communication. More specifically, node A does not hear node $\mathrm{E}$ when this node is forwarding a packet to node F. Based on the above observation, we can draw a conclusion that the overlap between two adjacent hops in the same route will not deteriorate the throughput. In this respect we consider two schemes, namely scheme-1 where the overlap between two adjacent hops in the same route is not counted into the overlap count, and scheme- 2 where the overlap between two adjacent hops in the same route is counted into the overlap count. For instance, in scheme-1 there is one overlap between hops of $\mathrm{A} \& \mathrm{~B}$ and $\mathrm{C} \& \mathrm{D}$ over the route $\mathrm{A} \rightarrow \mathrm{B} \rightarrow \mathrm{C} \rightarrow \mathrm{D}$ (see Fig. 1), whereas in scheme-2 there are 3 overlaps over the route $\mathrm{A} \rightarrow \mathrm{B} \rightarrow \mathrm{C} \rightarrow \mathrm{D}$, namely, the overlap between the hops of $\mathrm{A} \& \mathrm{~B}$ and $\mathrm{B} \& \mathrm{C}$, the overlap between the hops of A \& B and $\mathrm{C} \& \mathrm{D}$, as well as the overlap between the hops of B \& $\mathrm{C}$ and $\mathrm{C} \& \mathrm{D}$. Our simulation results in Fig. 5 demonstrate that scheme-1 is capable of attaining similar performance as scheme-2. This is a very important and useful merit, especially in multi-path routing scenarios, where multiple routes with zero overlap count are much easier to be found in scheme- 1 than in scheme-2.

The inter-path overlap count of multiple routes can be calculated by using the same method, where the position information of the nodes over multiple routes is taken into consideration. The intra-path overlap count and a positional information vector of the nodes along the route are inserted into the routing entry as shown in the Table I. Note that in the original DSR, since all the duplicated RREQs are discarded, it is unlikely to find the best route to destination. To avoid this kind of situation, intermediate nodes will forward the RREQs whose hop counts are not bigger than that of the previously received RREQs, even if they have the same ID. Therefore, the source node may re- 


\begin{tabular}{|l|l|l|l|l|}
\hline Destination & $\begin{array}{l}\text { Source } \\
\text { Route }\end{array}$ & $\begin{array}{l}\text { Positional } \\
\text { Information } \\
\text { Vector }\end{array}$ & $\begin{array}{l}\text { Hop } \\
\text { Count }\end{array}$ & $\begin{array}{l}\text { intra-path } \\
\text { Overlap } \\
\text { Count }\end{array}$ \\
\hline
\end{tabular}

TABLE I

ROUTING ENTRY STRUCTURE.

ceive multiple RREPs and hence obtain all possible routes to the destination.

It should be noted that in the route discovery process, the source node should be able to select best multiple routes from its route cache for data transmission. For instance, based on route selection metrics, it is possible that there would be too many potential routes from the source to the destination, especially in a high-density ad hoc network. For the sake of avoiding excessive overhead, a threshold is set in the destination node. When the number of the RREQs received by the destination is smaller than this threshold, the destination node will keep sending RREPs. Otherwise, the RREQs will be discarded. In our simulations, the threshold is set to 20 , which would be sufficiently large enough to find multiple routes with the fewest inter-path and intra-path overlaps (in our scenarios). In order to select the most suitable multiple routes from the route cache after receiving multiple RREPs from the destination node, three metrics are employed to measure the performance of selected routes as follows:

1) Hop Count;

2) Number of joint nodes between two nodes;

3) Inter-path and intra-path Overlap count over multiple routes.

Based on the information in Table I, the source node will select the most suitable multiple routes from its routing cache, according to the following rules:

1) The route with the smallest hop count in the source node's route cache has the highest priority to be selected as the first best route. If there are two or more routes with the same smallest hop count, then the intra-path overlap count is used to select a route with the fewest intra-path overlaps.

2) The second best route is selected from the remaining routes. Again, a route with a smaller hop count has higher priority to be selected.

3) If there are more routes with the same minimal hop count, the number of joint nodes will be used to select the second best route. Obviously, the route with smallest number of joint nodes will be selected as the second best route.

4) In the case that there are more routes with the same number of joint nodes, the overlap count (including intra-path and inter-path overlaps) of the current route and the first best route will be employed to decide the second best route.

5) Repeat steps 2), 3) and 4) to select the best remaining multiple routes

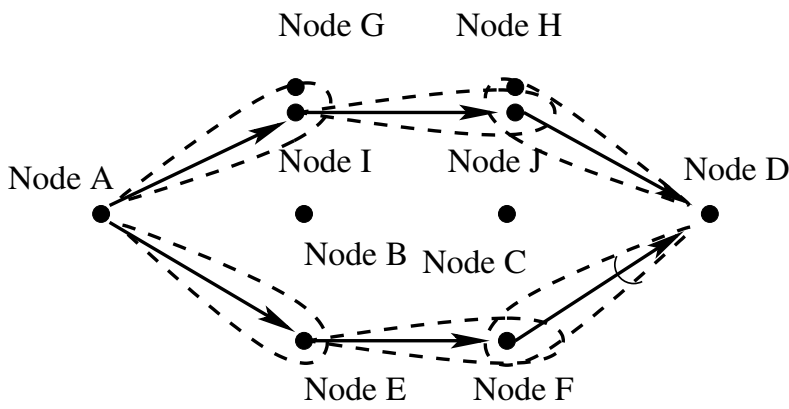

Fig. 2. An example of a DDSR-based two-path routing system.

\section{B. Data Transmission and Route Maintenance}

After the source selects the most suitable multiple routes to the destination, multiple data streams will be sent to the destination along these routes. An example of a DDSR-based two-path routing system is shown in Fig. 2, where nodes are capable of generating two beams at the same time, radiating to two different nodes. For example, node A simultaneously transmits packets to nodes $\mathrm{E}$ and $\mathrm{I}$ through transmit beams $\mathrm{A} \rightarrow \mathrm{E}$ and $\mathrm{A} \rightarrow \mathrm{I}$, respectively.

When a broken link (caused by mobility and packet loss) is detected by a node, an RERR message will be generated and sent in the upstream direction of the route. Once the source node receives the RRER packet, it removes the hop in error from its route cache and truncates all routes containing this hop. After assessing the number of active routes left in the route cache to the destination, the source node will carry out following steps: If there are two or more active routes left in the route cache, the source node will select the two best routes using the above-mentioned rules. Otherwise, the source node will initial a new route discovery process as discussed in Section II-A.

\section{Performance Results}

In this section the performance of the proposed DDSR-based multipath routing protocol is investigated by using our real-time network simulation testbed, where the IEEE 802.11 b standard is invoked. In the simulations, the input data generated at a Constant Bit Rate, is encapsulated into fixed 500 bytes UDP packets. In the physical layer, the receiver sensitivity is -93.0 $\mathrm{dBm}$, the IEEE $802.11 \mathrm{~b}$ data-rate is $2 \mathrm{Mbps}$ and the noise factor is 10.0. The directional antenna model employed in our simulations is capable of forming a sharp beam with a beamwidth of $2 \beta=40$ degrees, as portrayed in Fig. 3. The maximum antenna gain is $15.56 \mathrm{~dB}$, while the sidelobe gain outside the beam is $4.00 \mathrm{~dB}$. For simplicity, there is no fading in our simulation and free space is selected as the path loss model. Due to the space limitation, only a single-session scenario is considered in this paper, which means that there is only one source and one destination in the network. It will be extended to a multi-session scenario in our future research.

The performance of the proposed DDSR-based multi-path routing protocol is investigated in a random ad hoc network using directional antenna as seen in Fig. 4. Without loss of generality, single-path routing schemes and two-path routing schemes are studied in this figure. It is shown in Fig. 5 that the 


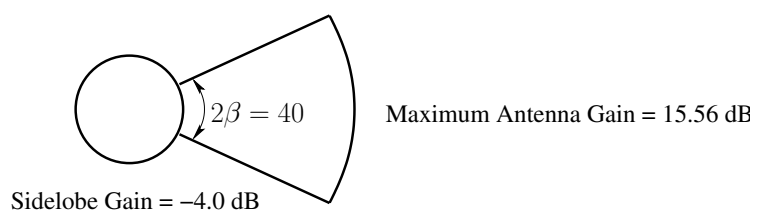

Fig. 3. Directional antenna model employed in our simulations.

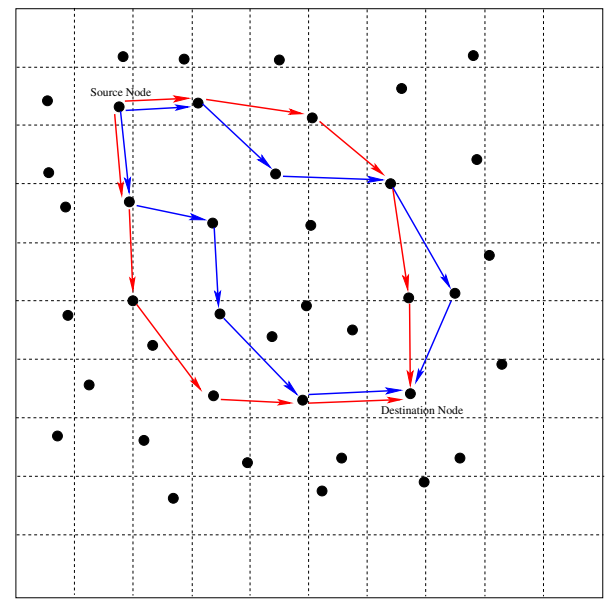

Fig. 4. Random ad hoc network. Two red routes are selected when using the DDSR-based multi-path routing protocol scheme-1, where overlaps between adjacent hops in the same route are not counted. By contrast, when invoking the DDSR-based multi-path routing protocol scheme-2, two blue routes will be used to transmit packets.

DDSR-based single-path routing protocol is capable of greatly improving the performance of the original DSR-based singlepath routing protocol, since there is no interference from the nodes hops away because of the directionality of the beam. The packet loss in the DDSR-based routing protocols occurs mainly because that when node $i$ is directionally communicating with node $i+1$, node $i$ can not receive data from node $i-1$. This kind of packet loss happens frequently when the input bit rate is higher than $600 \mathrm{Kbps}$. Based on this observation, a conclusion can be reached that we cannot further improve the performance by reducing overlaps between adjacent hops (in the same route). In other words, overlaps between adjacent hops in the same route cannot impact the throughput performance. Fig. 5 demonstrates that the DDSR-based single-path routing protocol (scheme-1) is capable of attaining a similar performance as the DDSR-based single-path routing protocol (scheme-2). It can also be seen from Fig. 5 that the DDSR-based two-path routing protocols further improve the throughput performance by simultaneously transmitting packets along two overlap-free paths. As shown in Fig. 5, two red routes are selected when using the DDSR-based multi-path routing protocol (scheme-1), where overlaps between adjacent hops in the same route are not counted. By contrast, when invoking the DDSR-based twopath routing protocol (scheme-2), two blue routes will be used to transmit packets. Again, the DDSR-based two-path routing protocol scheme-1 attains a similar performance as the DDSRbased two-path routing protocol scheme-2. Note that routes with zero overlap count are much easier to be found in scheme1 than in scheme-2. Scheme-1, however, has an advantage over
Random Network using Directional Antenna

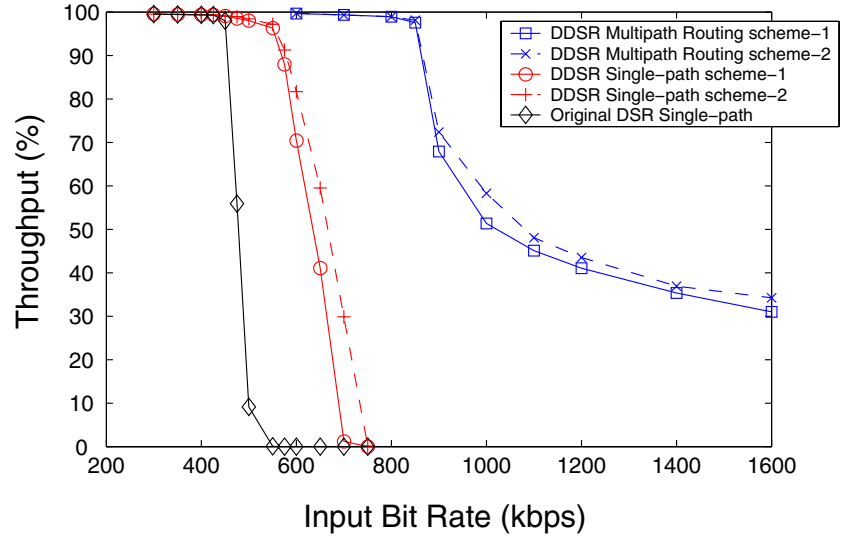

Fig. 5. Random ad hoc network using directional antenna. The proposed DDSR-based multipath routing protocol achieves the best performance, followed by the DDSR-based single-path routing protocol. The worst one is the original DSR-based single-path protocol.

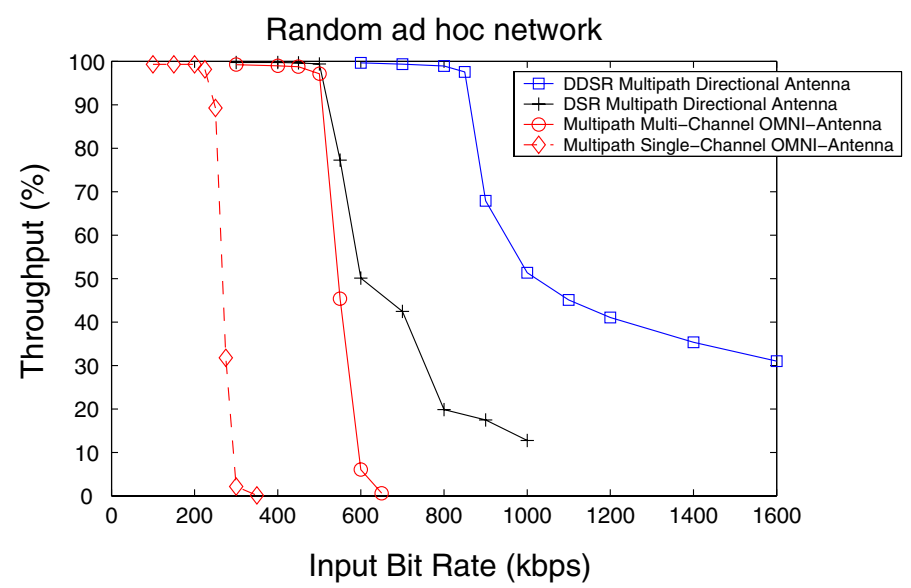

Fig. 6. Random ad hoc network. The proposed DDSR-based multipath routing protocol with directional antenna achieves considerable performance gains over other routing protocols, including the original DSR-based multipath routing protocol with directional antenna or omnidirectional antenna, as well as the multipath multichannel routing protocol with omnidirectional antenna.

scheme-2, especially in multi-path routing scenarios.

In Fig. 6, the performances of the proposed DDSR-based multipath routing protocol with directional antenna are compared with other routing protocols, including the original DSRbased multipath routing protocol with directional antenna discussed in [7], the multipath multichannel routing protocol with omnidirectional antenna described in [6], or the SMR scheme proposed in [3]. Without loss of generality, two-path routing schemes are considered in this figure. It should be noted that we have used a different transmit power for different routing schemes so that the average hop count of every path is 5. Obviously, the DDSR-based multipath routing protocol with directional antenna achieves the best performance, followed by the original DSR-based multipath routing protocol with directional antenna discussed in [7], in which case the inter-path and intrapath interferences are not eliminated as in the DDSR-based multipath routing protocol. The third scheme is the multipath 
multichannel routing protocol with omnidirectional antenna described in [6], where intra-path co-channel interference has not been suppressed. The SMR scheme proposed in [3] attains the worst performance since it can not reduce either intra-path or inter-path interference.

Finally, in Fig. 7 the proposed DDSR-based multipath routing protocol is examined in a dynamic ad hoc network environment, where nodes move randomly at a speed of $5 \mathrm{~m} / \mathrm{s}$. Again, the proposed DDSR-based multipath routing protocol achieves the best performance, since it is capable of eliminating both inter-path and intra-path interferences. Fig. 7 also indicates that the DDSR-based single-path routing protocol outperforms the original DSR-based multipath routing protocol, which means that the benefits of using multipath are counteracted by the inter-path interference and intra-path interference.

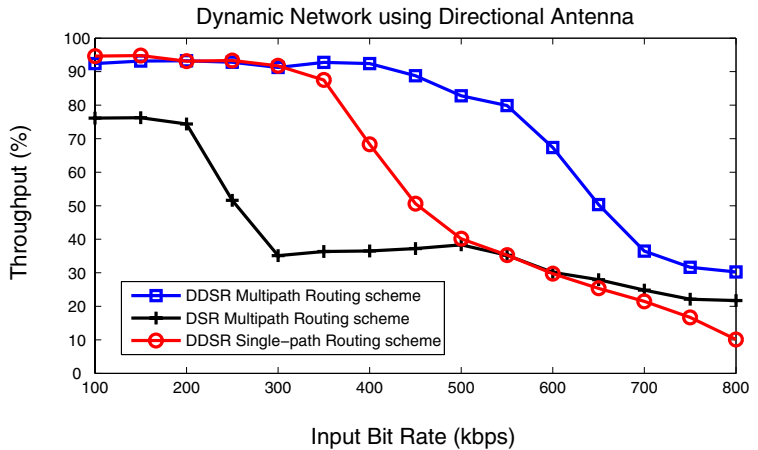

Fig. 7. Random dynamic ad hoc network. The proposed DDSR-based multipath routing protocol is investigated in a dynamic ad hoc network, where the nodes move randomly at a speed of $5 \mathrm{~m} / \mathrm{s}$.

\section{CONCLUSIONS}

In this paper we have proposed an DDSR-based multipath routing protocol, in order to enhance the performance of ad hoc networks using directional antennas. The proposed directional routing protocol aims at preventing interference from other nodes along the same path by exploiting the directionality of the beams and hence, improve the performance. Additionally, a multipath scheme is incorporated to further improve the throughput of the system by simultaneously transmitting packets over multiple paths. Finally, our comparative results demonstrated that the proposed DDSR-based multipath routing protocol with directional antenna is capable of achieving considerable performance gains over other routing protocols, including the original DSR-based multipath routing protocol with directional antenna discussed in [7], the multipath multichannel routing protocol with omnidirectional antenna described in [6], or the SMR scheme proposed in [3].

\section{REFERENCES}

[1] A. Nasipuri and S. R. Das, "On-demand multipath routing for mobile ad hoc networks," Proceedings of IEEE ICCCN'99, pp. 64-70, Oct. 1999.

[2] S. J. Lee and M. Gerla, "AODV-BR: backup routing in ad hoc networks," IEEE WCNC. 2000, vol. 3, pp. 1311-1316, Sept. 2000.

[3] S. J. Lee and M. Gerla, "Split multipath routing with maximally disjoint paths in ad hoc networks," ICC 2001, Vol. 10, pp. 3201-3205, June 2001.

[4] P. P. Pham and S. Perreau, "Performance analysis of reactive shortest path and multipath routing mechanism with load balance," Proceedings of IEEE INFOCOM 2003, Vol. 1, pp. 251-259, April 2003.

[5] S. Tsirigos and Z. J. Haas, "Multipath routing in the presence of frequent topological changes," IEEE Communications Magazine, Vol. 39, pp. 132138, Nov. 2001.

[6] B. Yan and H. Gharavi,, "Multi-path Multi-Channel Routing Protocol," IEEE International Symposium on Network Computing and Applications 2006, IEEE NCA06, Cambridge, MA USA, July 2006.

[7] S. Roy, S. Bandyopadhyay, T. Ueda, and K. Hasuike, "Multipath Routing in Ad Hoc Wireless Networks with Omni Directional and Directional Antenna: A Comparative Study," Proceedings of the 4th International Workshop on Distributed Computing, Mobile and Wireless Computing, pp. 184-191, 2001.

[8] S. K. Das, A. Mukherjee, S. Bandyopadhyay, K. Paul, and D. Saha, "Improving quality-of-service in ad hoc wireless networks with adaptive multi-path routing," Global Telecommunications Conference, 2000, vol. 1, pp. 261-265, 27 Nov.-1 Dec. 2000.

[9] J. H. Winters, "Smart antenna techniques and their application to wireless ad hoc networks," IEEE Wirelss Communications, vol. 13, pp. 77-83, August 2006.

[10] S. Yi, Y. Pei, and S. Kalyanaraman, "On the Capacity Improvement of Ad Hoc Wireless Networks Using Directional Antennas," Proc. Mobihoc'03, pp. 108-116, June 2003.

[11] R. Ramanathan, J. Redi, C. Santivanez, D. Wiggins, and S. Polit, "Ad hoc networking with directional antennas: a complete system solution," IEEE Journal on Selected Areas in Communications, vol. 23, pp. 496506, March 2005.

[12] R. R. Choudhury, X. Yang, R. Ramanathan, and N. H. Vaidya, "On designing MAC protocols for wireless networks using directional antennas," IEEE Transactions on Mobile Computing, vol. 5, pp. 477-491, May 2006.

[13] M. Takai, J. Martin, A. Ren, and R. Bagrodia, "Directional virtual carrier sensing for directional antennas in mobile ad hoc networks," Proc. Mobihoc'02, pp. 183-193, June 2002.

[14] M. Park, R. W. Heath, Jr., and S. M. Nettles, "Improving throughput and fairness for MIMO ad hoc networks using antenna selection diversity," Global Telecommunications Conference, 2004. GLOBECOM '04. IEEE, vol. 5, pp. 3363-3367, December 2004.

[15] D. B. Johnson and D. A. Maltz, "Dynamic Source Routing in Ad-Hoc Wireless Networks," Mobile Computing, T. Imielinski and H. Korth, Eds., Kluwer, pp. 153-181, 1996.

[16] H. Gharavi, "Control Based Mobile Ad-hoc Networks For Video Communications," IEEE Transactions on Consumer Electronics, vol. 52, No. 2, pp. 383-391, May 2006.

[17] H. Gharavi, "Multi-Channel Mobile Ad-hoc Links for Multimedia Communications," Proceedings of the IEEE, Vol. 96, No. 1, pp. 77-96, January 2008.

[18] B. Hu and H. Gharavi,, "DSR-Based Directional Routing Protocol for Ad Hoc Networks," IEEE Global Telecommunications Conference, 2007. GLOBECOM '07, pp. 4936-4940, Washington DC, USA, Nov. 2007. 